\title{
Imaging Grains and Grain Boundaries in Single-Layer Graphene: An Atomic Patchwork Quilt
}

Pinshane Y. Huang*, A.M. van der Zande*, C.S. Ruiz-Vargas*, W. S. Whitney*, M.P. Levendorf*, J. W. Kevek*, Y. Zhu*, J. Park*, P. L. McEuen*, D.A. Muller*

* Kavli Institute, Cornell University, Ithaca, NY 14853

Understanding the grain structure of single sheets of graphene is critical because grain boundaries are expected to significantly alter the electronic, magnetic, chemical, and mechanical properties of graphene monolayers [1-3]. Furthermore, single-layer graphene can be produced by chemical vapor deposition (CVD) on copper substrates [4] on up to meter scales[5], making their polycrystallinity almost unavoidable. By combining aberration-corrected scanning transmission electron microscopy (ADF-STEM) and dark-field transmission electron microscopy (DF-TEM), we image graphene grains and grain boundaries across six orders of magnitude in CVD graphene grown on copper. Combining these images with scanned probe and transport measurements, we probe the electrical and mechanical properties of the grain boundaries. These results, reported in Reference [6], enable studies on the structure, properties, and control of graphene grains and grain boundaries.

To characterize graphene membranes at all length scales, we first approached the atomic-scale using ADF-STEM imaging at $60 \mathrm{kV}$. The resulting images of graphene grain boundaries show every atom at a grain boundary and reveal that different grains stitch together predominantly via pentagonheptagon pairs. While the boundary dislocation resembles structures proposed theoretically [2], its aperiodicity contrasts with many of these models and will strongly affect the predicted properties of grain boundaries. We next used diffraction-filtered imaging to quickly and efficiently map the shape and orientation of several hundred grains and boundaries on the scale of tens of nanometers to tens of microns. While this method is usually applied to hundred-nanometer-thick foils, we report that remarkably, it also works on single-atom thick sheets when a low accelerating voltage is used. These images reveal an intricate patchwork of grains connected by tilt boundaries. We used these DF-TEM methods to correlate grain size with growth condition.

These simple methods to image grain structure enable direct studies of the physical properties of polycrystalline graphene. By correlating grain imaging with scanned probe and transport measurements, we show that grain boundaries dramatically weaken the mechanical strength of graphene membranes, but do not as dramatically alter their electrical properties. We first examined the failure strength of the polycrystalline CVD graphene membranes using atomic force microscopy (AFM). We used AFM phase imaging to image grains and then pressed downward with the AFM tip to test the mechanical strength of the membranes. We next probed the electrical properties of polycrystalline graphene by fabricating electrically-contacted devices using graphene from graphene grown with three different growth methods on copper. By comparing these measurements against corresponding DF-TEM images from each growth, we find that surprisingly, while mobility is clearly affected by growth conditions, high mobility does not directly correlate with large grain size. These methods will be crucial both for exploring synthesis strategies to optimize grain properties and for further studies on the microscopic and macroscopic impact of grain structure on graphene membranes[7]. 


\section{References}

[1] N. M. R. Peres, F. Guinea, A. H. Castro-Neto, Phys. Rev. B 73 ( 2006).

[2] O. V. Yazyev, S. G. Louie, Nature Mater., 9, 806-809 (2010).

[3] S. Malola, H. Hakkinen, P. Koskinen, Phys. Rev. B 81, 165447 (2010).

[4] X. Li et al., Science 324, 1312 (2009).

[5] S. Bae, et al. Nature Nanotechnol. 5, 574-578 (2010).

[6] P.Y. Huang, et al., Nature, 469, 389-392 (2011).

[7] This work was supported by the NSF through the CCMR and the NNI. Additional support was provided by the ARO and AFO, CONACYT-Mexico, DARPA-MTO, the MARCO Focused Research Center on Materials, Structures, and Devices. Ultrathin SiN grids supplied by SiMPore Inc. P.Y.H., A.M. vdZ, and C.S. R.-V. contributed equally.
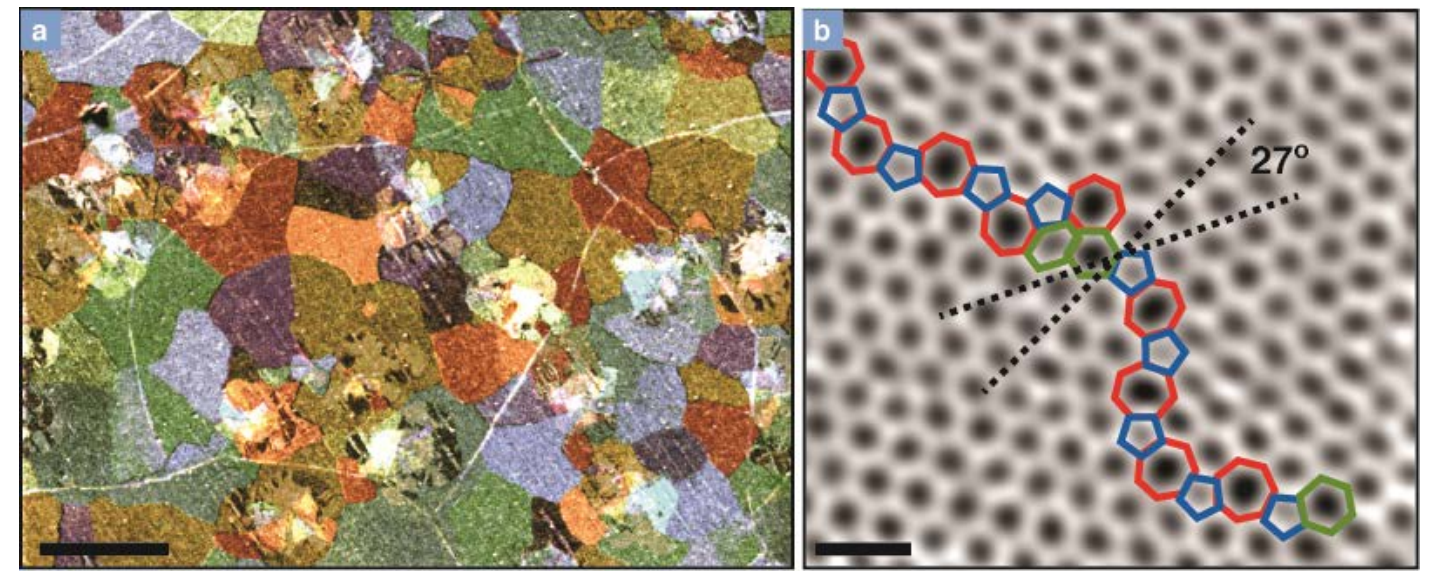

FIG. 1. Images of the grain structure of CVD graphene grown on copper. (a) Composite, false-color DF-TEM image of graphene grains imaged through 10-thick $\mathrm{nm}$ SiN membranes. Each color represents graphene of a small ( $\sim 5$ degrees) range of in-plane crystallographic orientations. Scale bar $2 \mu \mathrm{m}$. (b) ADF-STEM image of the atomic structure of a grain boundary in free-standing graphene. The two grains meet with a relative crystallographic rotation of $27^{\circ}$. Pentagons (blue), heptagons (red), and disordered hexagons (green) make up the meandering, a-periodic structure at the grain boundary. Reproduced from Reference [6], scale bar $5 \AA$.
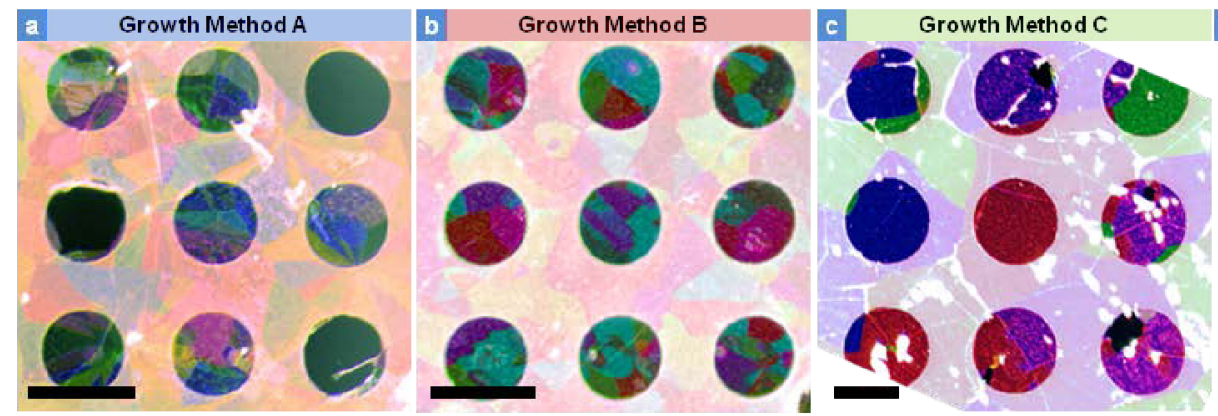

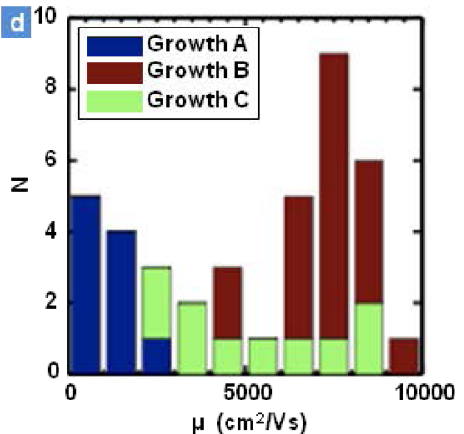

$\mu\left(\mathrm{cm}^{2} / \mathrm{N}_{\mathrm{s}}\right)$

FIG 2: a-c, Composite DF-TEM images of grain structure show variations with growth conditions. The graphene is suspended on perforated Quantifoil TEM grids and is still visible through the $20 \mathrm{~nm}$ $a$-C support film. Scale bars $2 \mu \mathrm{m}$. d, Vertically stacked histogram of room temperature mobilities measured from 50 devices using graphene Growth Methods A, B, and C of Reference [6]. Reproduced from Reference [6]. 